\title{
DRIVING LASER PULSE EVOLUTION IN A LASER WAKEFIELD ACCELERATOR
}

\author{
P. Volfbeyn, P. B. Lee, J. S. Wurtele, W. P. Leemans, Ernest Orlando Lawrence Berkeley National \\ Laboratory, University of California Berkeley, Berkeley, CA 94720, and G. Shvets, Princeton \\ Plasma Physics Laboratory, Princeton, NJ 08543
}

\begin{abstract}
A numerical study of the coupling between a high intensity laser pulse and a plasma wake is presented, in the context of laser wakefield acceleration in a hollow channel. One dimensional self-consistent equations are derived and numerically solved to provide a more detailed description of the laser pulse evolution for the case of propagation in a uniform plasma. The model obtained provides a simple method for inferring the plasma wake characteristics from measurement of changes in phase and amplitude of the driving laser pulse.
\end{abstract}

\section{INTRODUCTION}

Laser driven plasma accelerators, based on the excitation of extremely high longitudinal electric fields with phase velocity close to the speed of light [1] has received much theoretical and experimental attention for nearly two decades. A survey and comparison of different methods for laser acceleration and summaries of experimental and theoretical progress can be found in references [2] - [5]. In this paper we focus on the resonant Laser Wakefield Accelerator (LWFA), in which an ultra-short laser pulse $\left(\omega_{p} \Delta t \approx \pi\right.$, where $\Delta t$ is the laser pulselength , $\omega_{p}^{2}=\frac{4 \pi n e^{2}}{2 m_{0}}, \mathrm{n}$ is the local plasma density, $\gamma$ is the relativistic Lorentz factor, $e$ and $m_{0}$ are the charge and the rest mass of the electron) is used to excite a plasma wave via the ponderomotive force from the gradient in the laser pulse intensity.

Diffraction limits acceleration length, and hence the final energy of an accelerated charged particle in the LWFA to about one Rayleigh range. Laser guiding in plasma channels has been proposed as a means to extend the acceleration length. The index of refraction of a plasma with density $n$ can be approximated by $\eta \approx 1-\frac{\omega_{p}^{2}}{2 \omega^{2}}$, where $\omega$ is the laser frequency. Similar to an optical fiber, a plasma channel can provide optical guiding if the index of refraction peaks on axis. This requires a density profile that has a local minimum on axis. Guiding of laser pulses with moderate intensities (around
$10^{15} \mathrm{~W} / \mathrm{cm}^{2}$ ) over distances in excess of 20 Rayleigh ranges has been demonstrated experimentally [6].

For acceleration purposes, excitation of large amplitude plasma waves requires laser intensities on the order of $10^{18} \mathrm{~W} / \mathrm{cm}^{2}$ (for a laser wavelength $\sim 1$ micron). At such high intensities the index of refraction of the plasma is modified by its interaction with the laser pulse through relativistic effects as well as through a plasma density modulation arising from the excited plasma wave. The interaction with plasma affects both longitudinal and transverse properties of the laser pulse, which, in turn, leads to changes in the coupling between the laser pulse and the plasma as well as in properties of the accelerating plasma wave. These issues are important for evaluation and optimization of the efficiency of LWFA designs. In this paper a one dimensional (1-D), nonrelativistic analytic model of the driving pulse evolution and supporting simulation results will be presented, with emphasis on the effects of energy depletion, longitudinal laser pulse shape distortion, and changes in the group velocity and center wavelength of the pulse.

As in conventional fiber optics, the transverse characteristics of the laser beam are determined by the transverse profile of the guide, i.e. the plasma density profile. There are two main types of channels that have been considered in the literature, the parabolic channel and the hollow channel. The parabolic channel has a cylindrically symmetric, parabolic density profile with minimum density on axis. It is usually assumed that the plasma wake in a parabolic channel is purely electrostatic or, equivalently, that the channel size is large compared to the plasma wavelength [3], [7]. The hollow channel refers to a cylindrically symmetric step plasma density profile, where the density inside the channel is zero, and the wall thickness is much less than a plasma wavelength.

A hollow channel is particularly favorable for the propagation of a high quality electron beam. It supports a single electromagnetic plasma wave mode and can be designed to support only one guided laser mode [3], [8]. This suppresses the laser hose instability [3], [7]. Full control over the profile of the channels remains a major experimental challenge and density profiles are typically neither parabolic nor hollow. The transverse fields of the plasma wave excited in an arbitrary profile channel have not been found analytically [3], [9], although much pro-

\footnotetext{
${ }^{\dagger}$ Work supported by the US Department of Energy contracts FDDEFG-03-95ER-40936 and DE-AC03-76SF00098.
} 
gress has been made in numerically determining the electromagnetic plasma wake modes [10]. In what follows the analysis for the hollow channel case is presented. A similar treatment would be applicable to a channel with an arbitrary transverse plasma density profile, provided that the plasma wake fields were known.

In Sec. 2 the 1-D equations to model the evolution of the laser pulse in the plasma are derived and numerical solutions of these equations is presented. Conclusions and discussion comprise Sec. 3.

\section{SELF-CONSISTENT EVOLUTION OF THE LASER PULSE}

To study the effect of pump depletion on the driving laser pulse we start with the wave equation:

$$
\nabla \times \nabla \times \vec{a}_{0}+\frac{1}{c^{2}} \frac{\partial^{2} \vec{a}_{0}}{\partial^{2}}=\frac{4 \pi e}{m c^{3}} \vec{J}
$$

In the limit that the longitudinal velocity of the electrons remains much smaller than the speed of light and the transverse size of the laser pulse is much larger than the wavelength, the transverse canonical momentum of an electron moving in the laser fields is conserved; so that if the electrons are initially at rest $\gamma V / c=-a_{0}$, where $\mathrm{V}$ is the electron velocity, and $\gamma=1 / \sqrt{1-V^{2} / c^{2}}$. The transverse current density then is $\mathrm{J}=-\mathrm{nea}_{0} \mathrm{c}$ and

$$
\frac{4 \pi e}{m c^{3}} J=-\frac{\omega_{p}^{2}}{c^{2}} a_{0} \text {. }
$$

Assuming the vector potential is purely transverse, a transversely homogeneous plasma, and a plane laser wave and performing a change of variables $z=z, \xi=z-c t$ transforms Eq. (1) into

$$
\frac{\partial^{2} a_{0}}{\partial z^{2}}+2 \frac{\partial^{2} a_{0}}{\partial z \partial \xi}=k_{p}^{2} a_{0}
$$

Using $a_{0}=b e^{-i \Phi}$, where $\mathrm{b}$ is real and a slow function of $z$ and $\xi$, and $\Phi$ is also real and a slow function of $z$, (while $\Phi$ has fast variations in $\xi$ ), the real part of Eq. (3) becomes

$-\frac{\partial \Phi}{\partial(k z)} \frac{\partial \Phi}{\partial(k \xi)}=\frac{\chi}{2}$,

and the imaginary part is

$$
\frac{\partial}{\partial(k z)}\left(b \frac{\partial \Phi}{\partial(k \xi)}\right)=-\frac{\partial \Phi}{\partial(k z)} \frac{\partial b}{\partial(k \xi)},
$$

where equations were made dimensionless by dividing both parts by the wave number of the laser, $k^{2}$, and treating $\omega_{p} / \omega$ as a small quantity, Eqs. (4) and (5) contain only the lowest order in $\omega_{p} / \omega$ terms. Also, the quantity $\chi=k_{p}^{2} / k^{2}$ was introduced.
To simulate the effects of the laser pump depletion self-consistently we start with an expression for the plasma density modulation [14], obtained under assumption that the perturbation is small compared to the unperturbed density, $\delta n / n_{i}<<1$,

$\frac{\delta n}{n_{i}}(\xi)=\frac{b^{2}(\xi)}{4}+k_{p i} \int_{\xi}^{\infty} \sin \left(k_{p i}(\xi-\xi)\right) \frac{b^{2}(\xi)}{4} d \xi$

The right hand side of Eq. (4) can be rewritten as

$$
\frac{\chi}{2}=\frac{\omega_{p i}^{2}}{2 \omega^{2}}\left(1+k_{p i} \int_{\xi}^{\infty} \sin \left(k_{p i}(\xi-\xi)\right) \frac{b^{2}(\xi)}{4} d \xi\right) \text { (7) }
$$

to the lowest order in $b^{2}$, where the relativistic correction to the plasma frequency was taken into account.

The evolution of the amplitude $b$ and frequency $\frac{\partial \Phi}{\partial \xi}$

obtained by integrating Eqs. (4) and (5) numerically. Fig. 1 shows the longitudinal profile of the driving laser pulse vector potential and the relative change in the refractive index of the plasma, corresponding to the plasma wake.

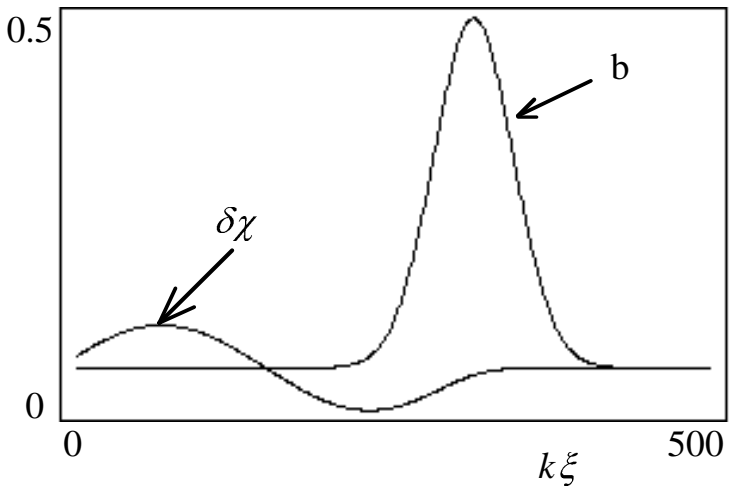

Figure 1. The amplitude of the laser pulse vector potential, $b$, gaussian pulse shape with the pulselength calculated to maximize the coupling to the plasma wake, and the plasma oscillation, $\delta \chi$, from Eq. (7). Plotted as function of distance from the head of the laser pulse, $k \xi$.

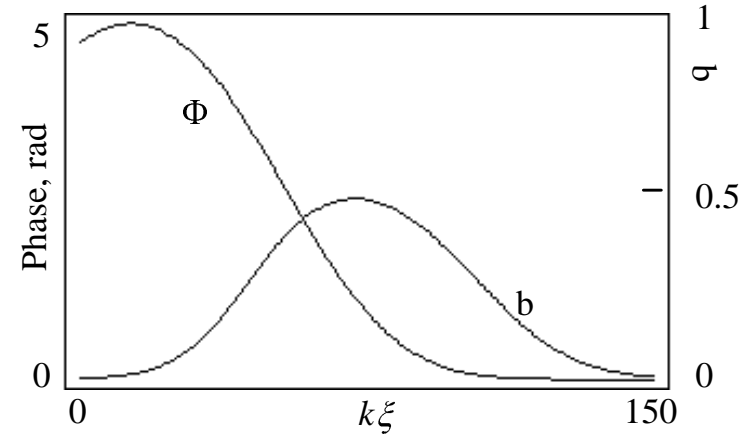

Figure 2. The amplitude of the laser pulse vector potential, $b$, and the phase, $\Phi$, as a function of $k \xi$ after propagating for one dephasing length. Calculated by integrating Eqs. (4) and (5) numerically with $\chi$ calculated from Eq. (7), with $\omega_{p} / \omega_{0}=1 / 50$, a laser pulse with a gaussian pulse shape with the pulselength 
calculated to maximize the coupling to the plasma wake, and amplitude $a_{0}=0.5$.

It is because different parts of the laser pulse experience different values of the index of refraction, the laser pulse develops a temporal phase chirp. In Fig. 2, $b$ and $\Phi$ are shown as functions of $\xi$ after the laser pulse propagated a distance $\mathrm{z}=\mathrm{L}_{\text {deph }}$.

The frequency spectrum of the laser pulse is redshifted and widened (Fig. 3a) resulting in a change in the laser pulse-shape (Fig. 3b).
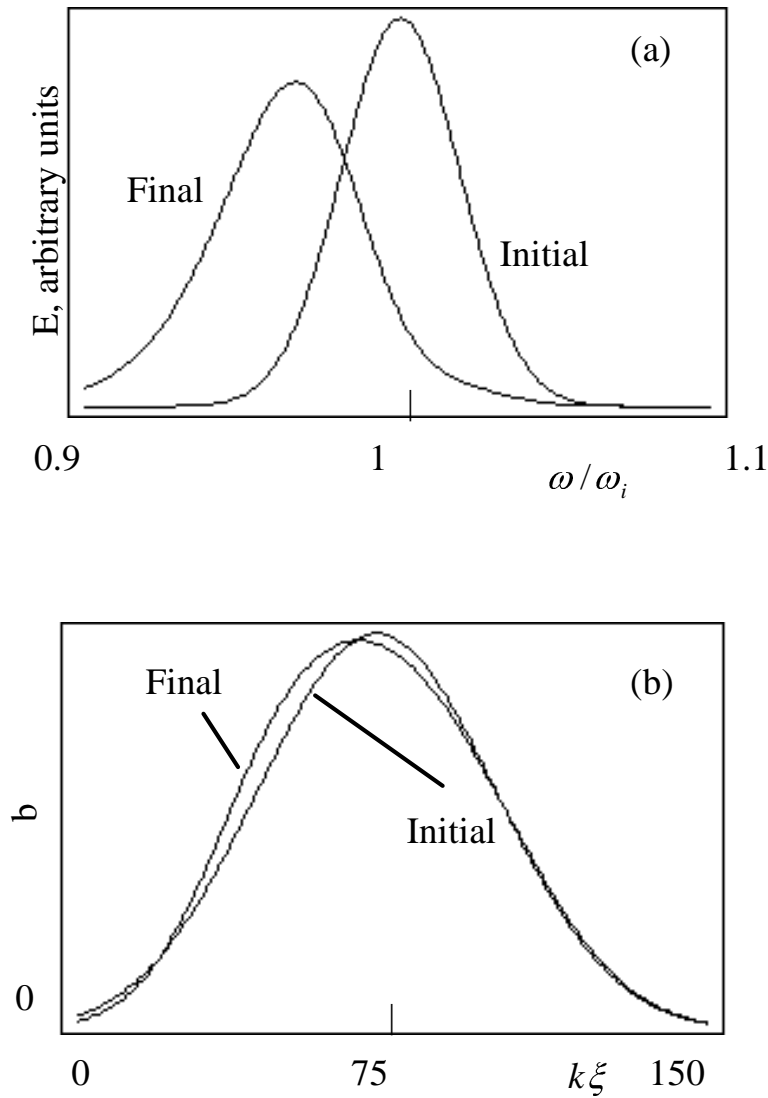

Figure 3. a). Spectrum of the laser pulse (unnormalized, arbitrary units) before and after propagating for one dephasing length. b). The amplitude of the laser pulse vector potential, $b$, before and after propagating for one dephasing length. Calculated by integrating Eqs. (4) and (5) numerically with $\chi$ calculated from Eq. (7), with $\omega_{p} / \omega_{0}=1 / 50$, a laser pulse with a gaussian pulse shape with the pulselength calculated to maximize the coupling to the plasma wake, and amplitude $a_{0}=0.5$.

The amplitude of the plasma wake remained nearly constant during the interaction.

\section{CONCLUSIONS}

A set of $1 \mathrm{D}$ equations for the evolution of a driving laser pulse in a homogeneous plasma was derived. The equations were solved numerically. The solutions demonstrated spectral red-shifting of the laser pulse. For realistic parameters, the shift in spectrum is quite pronounced. Measurement of the time resolved amplitude and phase of the driving laser pulse should supply valuable information about the plasma wake. The observed increase in the coupling strength supports the idea of trying to increase efficiency of a single stage accelerator by depleting the laser pulse energy, before dephasing terminates the acceleration.

\section{REFERENCES}

[1] T. Tajima and J.M. Dawson, "Laser electron accelerator", Phys. Rev. Lett., vol. 43, 267, (1979); Tajima, T. "Laser accelerator for ultra-high energies", Proc. 12th Int. Conf. on High-Energy Accelerators, Edited by: F. T. Cole and R. Donaldson, Batavia, IL, USA: Fermi Nat. Accelerator Lab, p. 470, 1983.

[2] E. Esarey, P. Sprangle, J. Krall, and A. Ting, "Overview of Plasma Based Accelerator Concepts", IEEE trans. on Plasma Science, v. 24, no. 2, p. 252, 1996.

[3] G. Shvets, "Interaction of intense lasers with plasmas", Ph.D. dissertation, MIT, 1995.

[4] C.E. Clayton, K. A. Marsh, A. Dyson, M. Everett, A. Lal, W. P. Leemans, R. Williams, and C. Joshi, Phys. Rev. Lett. 70, 37 (1993).

[5] N. E. Andreev, L. M. Gorbunov, V. I. Kirsanov, A. A. Pogosova, and R. R. Ramazashvili, "Resonant excitation of wakefields by a laser pulse in a plasma," JETP Lett., vol. 55, no. 10, pp. 571-576, 1992.

[6] C. G. Durfee III and H. M. Milchberg, Phys. Rev. Lett. 71, 2409 (1993).

[7] G. Shvets and J. S. Wurtele, "Instabilities of short pulse laser propagation through plasmas", Phys. Rev. Lett., vol. 73, p. 3540, 1994.

[8] T.C. Chiou, T. Katsouleas, C. Decker, W. B. Mori, G. Shvets, J. S. Wurtele, "Laser wakefield acceleration and optical guiding in a hollow plasma channel", Phys. Plasmas, vol. 2, pp. 310-318, 1995.

[9] G. Shvets , J. S. Wurtele, T. C. Chiou, and T. C. Katsouleas, "Excitation of Accelerating Wakefields in Inhomogeneous Plasmas", IEEE trans. on Plasma Science, v. 24, no. 2, p. 351, 1996.

[10] B. A. Shadwick, G. Shvets, J. S. Wurtele, Bull. Am. Phys. Soc., v. 41, p.1601.

[11] S. V. Bulanov, I. N. Inovenkov, V. I. Kirsanov, N. M. Naumova, and A. S. Sakharov, "Nonlinear depletion of ultrashort and relativistically strong laser pulses in an underdense plasma”, Phys. Fluids B 4 (7), July 1992.

[12] J. M. Rax and N. J. Fisch, "Nonlinear relativistic interaction of an ultrashort pulse with a cold plasma", Phys. Fluids B, vol. 4, pp. 1323-1331, 1992.

[13] W. Horton and T. Tajima, "Pump depletion in the plasmabeat-wave accelerator", Phys. Rev. A, vol.34, (no.5):411019, Nov. 1986.

[14] W.P. Leemans, C. W. Siders, E. Esarey, N. E. Andreev, G. Shvets, and W. B. Mori, "Plasma guiding and wakefield generation for second generation experiments", IEEE trans. on Plasma Science, v. 24, no. 2, p. 331, 1996. 